\title{
Ginsenoside Rg3 attenuates ischemia reperfusion induced renal injury in mice via induction of autophagy flux
} \author{
Dae Eun $\mathrm{Choi}^{2}$

\footnotetext{
${ }^{1}$ Department of Medical Science, Chungnam National University School of Medicine, Daejeon, Korea

${ }^{2}$ Department of Internal Medicine-Nephrology, Chungnam National University Hospital, Daejeon, Korea
}

Jin Ah Shin ${ }^{1}$, Jin young Jeong ${ }^{1}$, Haet Bit Hwang ${ }^{1}$, Soo hyun Han ${ }^{1}$, Eu Jin Lee ${ }^{1}$, Youngrok Ham ${ }^{1}, \mathrm{Ki}^{\mathrm{Rang}} \mathrm{Na}^{1}$, Kang Wook Lee${ }^{1}$,

Background: Ginsenoside Rg3 (Rg3) has been shown as protective effects via various mechanism. However, the reno-protective effect and the role of autophagy are not clearly evaluated. This study investigate Rg3 induces autophagy flux and reduces renal cell death in renal ischemia reperfusion injury (IRI).

Methods: C57BI/6 mice were divided into the following groups: sham; Rg3 treated sham; saline treated IRI mice; Rg3 treated IRI mice. Kidneys and blood were collected 24 hours after operation of mice (sham and IR operation). Renal function, kidney histology, and the protein expression of autophagy signals were evaluated.

Results: In IRI mice, the levels of blood urea nitrogen (BUN) and serum creatinine ( $\mathrm{SCr}$ ) were increased, compared to sham. The Rg3 treatment decreased the BUN and SCr in IRI mice. In addition, Rg3 treatment decreased the renal injury score including the renal tubular cell detachment and necrosis in IRI mice. Rg3 treated IRI mice showed significantly less oxidative stress and autophagy impairment, greater amounts of LC3 and Beclin-1, lower amounts of p62, and higher levels of renal ATP6E compared to saline treated IRI mice. Rg3 treatment also increased phosphorylation of AMPK in IRI mice kidney.

Conclusions: Rg3 has renoprotection against renal IR injury via enhancement of autophagy flux.

Corresponding author: Dae Eun Choi

E-mail: daenii@cnu.ac.kr

(c) The Korean Society for Transplantation

This is an Open Access article distributed under the terms of the Creative Commons Attribution Non-Commercial License (http://creativecommons.org/licenses/by-nc/4.0/) which permits unrestricted non-commercial use, distribution, and reproduction in any medium, provided the original work is properly cited. 